\title{
Oral health related behaviours reported by elite and professional athletes
}

\begin{abstract}
Background

In elite sport, protection of an athlete's health is a priority, however research indicates a substantial prevalence of oral disease in elite and professional athletes. The challenges to oral health from participation in sport require investigation to identify effective strategies and mitigate risk.
\end{abstract}

\section{Aim}

To explore athlete-reported oral health behaviours, risks and potential for behaviour change in a representative sample of elite athletes based in the UK.

\section{Method}

This was a cross sectional study. We provided oral health screening for 352 elite and professional athletes from June 2015-September 2016; 344 athletes also completed a questionnaire.

\section{Results}

Median age was 25 years (range 18 - 39) and 236 (67\%) were male. 323 (94.2\%) said they brush twice daily. $136(40 \%)$ said their most recent dental attendance was within the previous 6 months. 97 (28\%) would be assessed as high consumers of sugar in their regular diet. Use of sports nutrition products was common; $288(80 \%)$ reported using sports drinks during training or competition but were positive about behaviour changes.

\section{Conclusion}

Despite reporting positive oral health related behaviours, athletes have substantial amounts of oral disease. Athletes are willing to consider behaviour change related to daily plaque removal, increased fluoride availability and regular dental visits to improve oral health.

\section{Key Points}

- Oral diseases and athlete-reported negative performance impacts are common in elite and professional athletes

- Advises dentists that elite athletes are willing to adopt oral health related behaviours to mitigate the risks to oral health associated with sport

- Dentists should be aware of the need for enhanced prevention for sports people 


\section{Introduction}

In elite sport, protection of an athlete's health is a priority, however dental caries, periodontal diseases and erosive tooth wear (ETW) remain prevalent. ${ }^{1-4}$ Furthermore, athletes with poor oral health report negative impacts on wellbeing, quality of life, training and performance. ${ }^{1-4}$ Severe events such as acute dental infections or oro-facial trauma, leading to time lost from training and even competition, occur infrequently. However chronic impacts which may not lead to time loss, but rather a reduction in quality of training, are commonly reported, and at elite level may have important consequences. 4,5 Likely challenges to oral health in sport include increased risk of dental caries due to increased and/or inappropriate consumption of dietary carbohydrates within usual diet or sports nutrition products such as sports drinks, and energy bars or gels. ${ }^{6,7}$ These products are marketed with no accompanying guidance related to oral health. Sports drinks tend to be acidic, therefore may also contribute to ETW. ${ }^{8}$ Lack of awareness or prioritisation may also be a factor in elite sport. ${ }^{9}$ The risks of oral disease may be further increased due to alterations in saliva composition during exercise ${ }^{10}$ and immune suppression following intense effort. ${ }^{11}$ Effective oral health promotion strategies may minimise performance impacts from poor oral health. ${ }^{4}$ Although there is good evidence for oral health promotion and prevention outside of sport, ${ }^{12}$ the challenges to oral health from sport and those related to implementation in this environment, confer unique characteristics that require investigation to identify effective strategies and mitigate risk. ${ }^{13}$

\section{Aims}

The aim of this study was to explore athlete-reported oral health behaviours, risks to oral health and potential for behaviour change in a representative sample of elite athletes.

\section{Methods}

\section{Study design}

This was a cross-sectional study, conducted at UK elite athlete training centres between June 2015 and August 2016. We provided oral health screening for 256 athletes on podium potential/ placement programmes for the 2016 Rio Olympic Games and 96 professional athletes (352 in total) across 11 sports which we categorised as "endurance", "strength and power"(events lasting less than two minutes) or "mixed" (e.g. team sports). The methods and results are reported in a previous paper. ${ }^{4}$ The athlete-reported data presented in this paper were collected with a self-administered questionnaire, completed at the screening appointment.

\section{Eligibility criteria}

- Member of elite (Olympic or professional) training/development squad

- Aged 18 years or over

- Able to understand the consent process with the aid of a translator if required 
- Able to understand and complete the questionnaire with the aid of a translator if required Ethical approval was received from University College London research ethics committee (Project ID $6388 / 001)$. Informed written consent was obtained. Participation in the study was entirely voluntary and with no obligation.

\section{Athlete-reported oral health behaviours, risks to oral health and potential for behaviour change}

The questionnaire was developed with input from our advisory group which included academics, athletes, and dentists with an interest in sport. Items to explore self-reported oral health related behaviours were based on those used in the Adult Dental Health (ADH) Survey 2009. ${ }^{14}$ They included: frequency of tooth brushing, use of additional oral hygiene methods, most recent dental attendance, type of service used, most important factor when making a dental appointment and previous dental advice received. Use of additional oral hygiene aids included electric toothbrush (ETB),fluoride mouthwash, interdental cleaning and sugar free chewing gum (SFG). Risks to oral health included tobacco use and consumption of sugar in usual diet. The Scientific Advisory Committee on Nutrition (SACN) advises that the maximum free sugar consumption for the UK diet should be $5 \%{ }^{15}$ Using the method described in ADH 2009, athletes who indicated that they consumed a serving of cakes, or sweets, or soft drinks six or more times a week were categorised as high sugar consumers. ${ }^{14}$ We also explored knowledge of risks to oral health of sports nutrition products (SNPs). We also asked about athletes' use of SNPs before, during and after training and competition. Finally, we asked which behaviours athletes would consider adopting if it would improve their oral health. The research advisory group reviewed the questionnaire which was piloted before use.

\section{Statistical analysis}

We used a standard statistical package (IBM SPSS Statistics for Windows, Version 22.0. Armonk, NY). For data analysis. Counts and percentages summarised the categorical data. This report was guided by the STROBE statement of observational studies ${ }^{16}$.

\section{Results}

Median age was 25 years (range 18 - 39) and 236 (67\%) were male. The demographic characteristics of the group are presented in Online table 1. There were 50 (14.2\%) athletes in the strength and power (S\&P) category, 143 (40.6\%) in the endurance category and 159 (45.2\%) in the mixed category. Table 1 summarises the prevalence of oral disease in each category and sport. Questionnaire data were available for up to 344 athletes (50 (14.5\%) S\&P, 140 (40.7\%) endurance and 154 (44.8\%) mixed). Eight questionnaires were not returned due to time constraints and some athletes omitted some response options. 


\section{Oral health behaviours (Online Tables 2, 3 and 4)}

Overall, 323 (94.2\%, 95\% Cl 91.8-96.7) reported brushing their teeth at least twice daily. Regarding use of additional methods for oral hygiene, $190(55.9 \%, 95 \% \mathrm{Cl} 49.9-60.4)$ said they used an ETB, 148 (43.7\%, (95\% Cl 37.9-48.3) said they used dental floss or interdental brushes, 139 (40.9\%, 95\% Cl 35.445.6) said they used fluoride mouthwash and 120 (35.1\%, 95\% $\mathrm{Cl} 30.32-40.3)$ reported using sugar free chewing gum (SFG). Three hundred and twelve $(90 \%, 95 \% \mathrm{Cl}(87.1-93.4)$ athletes reported drinking water on at least six days or more per week.

\section{Risks to oral health (Online Tables 5, 6 and 7)}

One $(0.3 \%)$ athlete reported current use of smokeless tobacco. We categorised $97(28.2 \%, 95 \% \mathrm{Cl}$ 23.7-33.2) of athletes as high consumers of sugar in their regular diet. Regarding the use of sports nutrition products, $28885.7 \%$ (95\% Cl 81.5-89.1) of the 336 athletes who provided this information reported using sports drinks at least sometimes during training/competition, 198 (58.8\%, 95\% Cl 53.463.9) energy bars and $239(70.3 \%, 95 \% \mathrm{Cl} 65.9-75.5)$ energy gels.

\section{Dental service considerations (Online Tables 8, 9, 10 and 11)}

We asked how recently athletes had attended a dentist. Fewer than half $(136,39.5 \%, 95 \% \mathrm{Cl} 34.5-$ 44.8) of the athletes said they had attended for a dental visit within the previous 6 months. Three quarters $(262,76.2 \%, 95 \% \mathrm{Cl} 71.4-80.4)$ said they recalled receiving oral hygiene advice from a dental professional at some time and just over half $(206,59.9 \%, 95 \% \mathrm{Cl} 54.6-64.9)$ said they recalled receiving advice about diet. When asked what type of dental service they used for their most recent dental visit 141 (41\%, 95\% Cl 35.9-46.3) said an NHS dentist, 147 (45.6\%, 95\% Cl 40.5-50.9) said a private dentist and 34 (9.9\%, 95\% Cl 7.13-13.52) said a private dental hygienist. Athletes were asked which single factor was the most important when arranging a dental appointment. However, twenty indicated more than one factor. Of the remaining 314, 195 (62.1\%, 95\% Cl 56.6-67.3) said convenience, 85 (27.1\%, 95\% Cl 22.5-32.3) reputation of the dentist and 34 (10.8\%, 95\% Cl 6.9-13.2) cost.

\section{Oral health beliefs and potential for behaviour change}

The majority of athletes recognised that smoking and sugary foods and drinks including sports nutrition products could damage oral health (Table 2). The potential for behaviour change options and athlete responses are listed in Table 3. With the exception of reducing snacking between meals most athletes were positive about potential for behaviour change.

\section{Discussion}

\section{Key findings}

This is the first study to investigate self- reported oral health behaviour and challenges to oral health in representative samples of elite and professional athletes from different sports. In general, athletes 
report favourable oral health behaviours including toothbrushing before bed and in the morning but fewer than half had attended a dentist within the previous six months. Overall, we categorised $28.2 \%$ of athletes as high consumers of sugar in their regular diet, however $58.8 \%$ reported using energy bars and $70.3 \%$ energy gels during training and competition and fewer than half $(46 \%)$ athletes said they could or probably could reduce snacking between meals. Although $85.7 \%$ reported using sports drinks at least sometimes during training/competition, $80.4 \%$ said they could or probably could reduce sugary drinks including sports drinks between meals. Athletes said that they would consider regular dental visits, use of additional oral hygiene aids and increasing fluoride availability to improve oral health.

\section{Strengths and limitations of the data}

The strength of this study is the number of participants and completeness of the sample screened in each sport. Conducting studies in elite sport is difficult due to the competing pressures for time on the athletes and their support teams. However, self-reported measures can be unreliable and only serve as a proxy measure for oral hygiene and dietary habits. The questionnaire was completed independently and anonymously for convenience and to limit responder bias. However, it provided limited information regarding whether the athletes used sports drinks, energy bars and gels on the advice of coaches and/or nutritionists, or if they used them in response to marketing/ availability. Information on the content of the snacks consumed by athletes was also limited. Use of qualitative methods such as interviews or focus groups would have yielded a much greater depth of information but would have required a greater time commitment from the athletes which was not possible during this study.

\section{Comparison with other studies}

Few studies have investigated oral health behaviours and risks to oral health in this relatively young adult group. ${ }^{17-19}$ A cautious comparison may be made with adults of a similar age in the general population in England, Wales and Northern Ireland. ${ }^{19}$ Elite athletes report more favourable oral health behaviours; $94 \%$ compared to $74 \%$ say they brush morning and night and $43 \%$ compared to $18 \%$ say they use dental floss or interdental brushes. Only one athlete reported currently using smokeless tobacco (the proportion of smokers in the general population is around $28 \%$ ) and $28.2 \%$ compared to $55 \%$ would be classed as high consumers of sugar in their general diet. The findings from this study support those from one study of Nigerian college athletes ${ }^{17}$ and another study limited to triathletes ${ }^{18}$ which concluded by recommending "raising athletes' awareness of their specific increased risk for dental caries and erosion and demonstrating how to optimize their oral hygiene and advice". 


\section{Evidence-based interventions to improve oral health}

We found differences in prevalence of oral diseases between different sports. However the document "Delivering better oral health; an evidence-based toolkit" (DBOH) recommends that everyone should be given the benefit of advice and support to change behaviour regarding their general and dental health, not just those thought to be 'at risk'. ${ }^{12}$ Therefore strategies identified in this paper are appropriate to all sports

\section{Reducing risks to oral health from lifestyle}

Athletes reported high consumption of energy gels and bars during training and competition despite believing that they can damage oral health. However fewer than half felt they could reduce snacking. Hydration is an important consideration in sport ${ }^{20}$ and most athletes reported using sports drinks however, many felt that they could reduce their intake of sports drinks. The use of beverages and supplements containing sugars should be discouraged. ${ }^{7}$ Many rowers regularly drink sugar free squash, however this is not associated with increased caries or ETW in this group, therefore could be a useful alternative to proprietary sports drinks for hydration. For post-event hydration, milk could be substituted for proprietary sports drinks, and plain water is adequate if combined with electrolyte and carbohydrate-containing foods such as those normally eaten during the recovery period. 7,21,22 Many athletes (83.2\%) would consider the use of SFG but DBOH does not recommended it as a preventive adjunct. There is however some evidence that it may have a potential role in caries prevention. ${ }^{23}$ Athletes reported receiving oral hygiene advice and advice about diet from a dental professional at some time and dental professionals are well placed to identify potential lifestyle problems such as eating disorders. ${ }^{13}$ Sport nutrition is one of the cornerstones for athlete preparation and therefore well placed to deliver benefits across performance, general health, oral health and wellbeing. It would therefore make sense for strategies incorporating oral health to be jointly developed by registered sport nutritionists, oral health experts and other athlete support team members. ${ }^{7}$

\section{Improving oral health through oral health screening and coaching}

Oral health promotion in sport is most likely to be successful if it is embedded within overall athlete general health and performance promotion ${ }^{24}$ but most members of the athlete support team are not expert in this area and therefore specialist input is needed. Coaches, nutritionists and those who work with athletes should have robust training to ensure that athletes balance performance with their oral and general health. Dental recall intervals are based on risk ${ }^{25}$ and regular attendance for dental checks does not necessarily predict better oral health. ${ }^{26}$ However regular checks are important, not only to identify oral diseases at an early stage but also as an opportunity to enhance motivation towards a high standard of oral health. ${ }^{27}$ Most athletes said they would attend for regular dental checks if it would improve their oral health, but convenience is an important consideration. Oral diseases such as 
caries, ETW and periodontal diseases do not present with pain in their early stages and athletes may delay a dental check or even treatment until they perceive that they have a problem. Athletes may not be brushing effectively as most had evidence of gingival inflammation or risk factors present. Interdental cleaning using dental floss or other methods is important for optimum oral health. ${ }^{12,28}$ Although less than half currently do this, most athletes said they would consider cleaning interdentally if it would improve oral health. However practical instruction is required to improve oral hygiene skills. Our high recruitment rate of athletes underlines the importance of providing screening and simple oral health promotion/preventive advice at athletes' training centres, preferably combined with education and coaching in practical oral hygiene skills.

\section{Opportunities to mitigate risk through increased fluoride availability}

The most important behavioural factor affecting both caries and periodontal health is routinely performed oral hygiene with fluoride. ${ }^{29}$ When normal strength fluoride toothpaste (1000-1450ppm) is used, fluoride mouthwash used at a different time to brushing can be advised ${ }^{30}$. Many athletes said they could use a fluoride mouthwash, at a different time to brushing, therefore this could be an opportunity to increase fluoride availability but requires a new behaviour. Where caries risk is increased, higher strength prescription fluoride $(2800 \mathrm{ppm})$ is indicated ${ }^{31}$. Very high strength fluoride toothpaste $(5000 \mathrm{ppm})$ may also have a protective effect against erosion ${ }^{32}$. Nearly all athletes said they brushed in accordance with the widely recognised oral health advice of twice daily ${ }^{12}$, therefore using prescription strength fluoride toothpaste would increase fluoride availability without requiring change in current behaviour.

\section{Enhancing oral health related behaviour through behaviour change techniques}

To date, there is no evidence show which behaviour change technique (BCT) is best for enhancing health behaviours related to oral health. ${ }^{33}$ However, the current dominant approach to understanding health behaviour is the COM-B model ${ }^{34}$ and it has been suggested that interventions based on this behaviour change theory may be successful. ${ }^{35}$ Further research including consultation with all stakeholders including sports nutritionists, sport and exercise medicine practitioners and dental professionals is needed to ensure quality and relevance within elite athlete care.

\section{Conclusion}

Elite and professional athletes report more favourable oral health behaviours but still have similar levels of oral disease to the general population. Athletes say they would consider simple behaviour change including reduction in the use of sports drinks, attendance for regular screening and adoption of additional oral hygiene methods. These findings help inform on the design of interventions to improve/maintain oral health and reduce performance impacts. 


\section{Declaration of interests}

This project was jointly funded by a grant from GSK and UCL IMPACT (award number 157871). The authors declare no conflict of interest. The Centre for Oral Health and Performance is part of the UK IOC Research Centre for Prevention of Injury and Protection of Athlete Health with the Institute of Sport Exercise and Health (ISEH) and the National Centre for Sport and Exercise Medicine (NCSEM).

\section{Acknowledgements}

We wish to acknowledge the input from our advisory group, the athletes and support staff from all the sports who so generously gave of their time to contribute to this research project. Thank you also to Miss Sarah Needleman and Mrs Karen Wigmore for data entry and to Mrs Karen Wigmore, Mrs Alison O'Neil and Miss Laura Wigmore for assistance during data collection. We are grateful to Professor Susan Michie and Professor Robert West of the UCL Centre for Behaviour change for advice regarding the design of the behaviour change elements of the questionnaire. 


\section{Reference list}

1. Ashley P, Di lorio A, Cole E, Tanday A, Needleman I. Oral health of elite athletes and association with performance: a systematic review. Br J Sports Med. 2015;49(1):14-19.

2. Needleman I, Ashley P, Meehan L, et al. Poor oral health including active caries in 187 UK professional male football players: clinical dental examination performed by dentists. $\mathrm{Br} \mathrm{J}$ Sports Med. 2016;50(1):41-44.

3. Needleman I, Ashley P, Petrie A, et al. Oral health and impact on performance of athletes participating in the London 2012 Olympic Games: a cross-sectional study. Br J Sports Med. 2013;47(16):1054-1058.

4. Gallagher J, Ashley P, Petrie A, Needleman I. Oral health and performance impacts in elite and professional athletes. Community Dent Oral Epidemiol. 2018;46(6):563-568.

5. Engebretsen L, Soligard T, Steffen $\mathrm{K}$, et al. Sports injuries and illnesses during the London Summer Olympic Games 2012. Br J Sports Med. 2013;47(7):407-414.

6. Moynihan PJ, Kelly SA. Effect on caries of restricting sugars intake: systematic review to inform WHO guidelines. J Dent Res. 2014;93(1):8-18.

7. Needleman I, Ashley P, Fairbrother T, et al. Nutrition and oral health in sport: time for action. Br J Sports Med. 2018;52(23):1483-1484.

8. Lussi A, Jaeggi T, Zero D. The role of diet in the aetiology of dental erosion. Caries Res. 2004;38 Suppl 1:34-44.

9. Needleman I, Ashley P, Fine $P$, et al. Consensus statement: Oral health and elite sport performance. Br Dent J. 2014;217(10):587-590.

10. Mulic $A$, Tveit $A B$, Songe $D$, Sivertsen $H$, Skaare $A B$. Dental erosive wear and salivary flow rate in physically active young adults. BMC Oral Health. 2012;12:8.

11. Gleeson M. Immune function in sport and exercise. J Appl Physiol. 2007;103(2):693-699.

12. Public Health England. Delivering better oral health: an evidence-based toolkit for prevention Third edition 2017; www.gov.uk/government/publications/delivering-betteroral-health-an-evidence-based-toolkit-for-prevention. Accessed 18th Jan 2018.

13. Needleman I, Ashley P, Fine P, et al. Oral health and elite sport performance. Br J Sports Med. 2015;49(1):3-6.

14. O Sullivan I, Lader D, Beavan-Seymour C, Chenery V, Fuller E, Sadler K. Foundation Report: Adult Dental Health Survey 2009 (Technical information). In: care TNICfhas, ed. 1 ed2011.

15. SACN. Carbohydrates and Health. London: Scientific Advisory Committee on Nutrition;2015.

16. von Elm E, Altman DG, Egger M, et al. The Strengthening the Reporting of Observational Studies in Epidemiology (STROBE) statement: guidelines for reporting observational studies. PLoS Med. 2007;4(10):e296.

17. Azodo CC, Osazuwa O. Dental conditions among competitive university athletes in Nigeria. Odontostomatol Trop. 2013;36(141):34-42.

18. Bryant S, McLaughlin K, Morgaine K, Drummond B. Elite Athletes and Oral Health. Int J Sports Med. 2011;32(9):720-724.

19. Chadwick B, White D, Lader D, Pitts N. 5: Preventive behaviour and risks to oral health - a report from the Adult Dental Health Survey 2009. In: The Health and Social Care Information Centre.; 2011.

20. Pegoretti C, Antunes AEC, Manchado-Gobatto FdB, Capitani CD. Milk: An Alternative Beverage for Hydration? Food and Nutrition Sciences. 2015;06(06):547-554.

21. Shirreffs SM, Sawka MN. Fluid and electrolyte needs for training, competition, and recovery. J Sports Sci. 2011;29 Suppl 1:S39-46.

22. Needleman I, Ashley P, Fine P, et al. Infographic: oral health in elite athletes. Br J Sports Med. 2017;51(9):757.

23. Twetman S. Consistent evidence to support the use of xylitol- and sorbitol-containing chewing gum to prevent dental caries. Evid Based Dent. 2009;10(1):10-11. 
24. Dijkstra HP, Pollock N, Chakraverty R, Alonso JM. Managing the health of the elite athlete: a new integrated performance health management and coaching model. Br J Sports Med. 2014;48(7):523-531.

25. NICE. Dental checks: intervals between oral health reviews. In. nice.org.uk/guidance/cg19, trans. London2004.

26. Hill KB, Chadwick B, Freeman R, O'Sullivan I, Murray JJ. Adult Dental Health Survey 2009: relationships between dental attendance patterns, oral health behaviour and the current barriers to dental care. Br Dent J. 2013;214(1):25-32.

27. Needleman I, Ashley P, Weiler R, McNally S. Oral health screening should be routine in professional football: a call to action for sports and exercise medicine (SEM) clinicians. $\mathrm{Br} J$ Sports Med. 2015.

28. European Federation of Periodontology. EFP provides clear guidance to public on interdental cleaning in response to media controversy about flossing. 2016; www. efp.org/efp-guidanceon-interdental-cleaning. Accessed 7th March 2018, 2018.

29. Jepsen S, Blanco J, Buchalla W, et al. Prevention and control of dental caries and periodontal diseases at individual and population level: consensus report of group 3 of joint EFP/ORCA workshop on the boundaries between caries and periodontal diseases. $J$ Clin Periodontol. 2017;44 Suppl 18:S85-s93.

30. Marinho VC, Chong LY, Worthington HV, Walsh T. Fluoride mouthrinses for preventing dental caries in children and adolescents. Cochrane Database Syst Rev. 2016;7:CD002284.

31. Walsh T, Worthington HV, Glenny AM, Appelbe P, Marinho VC, Shi X. Fluoride toothpastes of different concentrations for preventing dental caries in children and adolescents. Cochrane Database Syst Rev. 2010(1):CD007868.

32. Pretty IA. High Fluoride Concentration Toothpastes for Children and Adolescents. Caries Res. 2016;50 Suppl 1:9-14.

33. Asimakopoulou K, Newton JT. The contributions of behaviour change science towards dental public health practice: a new paradigm. Community Dent Oral Epidemiol. 2015;43(1):2-8.

34. Michie S, Stralen MMv, West R. The behaviour change wheel: A new method for characterising and designing behaviour change interventions. Implemetation Science. 2011;6(42).

35. Newton JT, Asimakopoulou K. Minimally invasive dentistry: Enhancing oral health related behaviour through behaviour change techniques. Br Dent J. 2017;223(3):147-150. 